\title{
Evaluation of Two Crew Module Boilerplate Tests Using Newly Developed Calibration Metrics
}

\author{
Lucas G. Horta and Mercedes C. Reaves
}

\begin{abstract}
1 Abstract
The paper discusses a application of multi-dimensional calibration metrics to evaluate pressure data from water drop tests of the Max Launch Abort System (MLAS) crew module boilerplate. Specifically, three metrics are discussed: 1 ) a metric to assess the probability of enveloping the measured data with the model, 2) a multi-dimensional orthogonality metric to assess model adequacy between test and analysis, and 3) a prediction error metric to conduct sensor placement to minimize pressure prediction errors. Data from similar (nearly repeated) capsule drop tests shows significant variability in the measured pressure responses. When compared to expected variability using model predictions, it is demonstrated that the measured variability cannot be explained by the model under the current uncertainty assumptions.
\end{abstract}

\section{Introduction}

During the development of the Apollo command module (CM), many studies were conducted to understand the behavior of the CM upon returning to Earth. Because of the limited computational capabilities at the time, engineers made extensive use of experimental data to supplement the relatively simple analyses. These studies were performed to understand the landing behavior under a variety of conditions. Interestingly, Apollo was initially to include a land-landing architecture. Thus many of the early studies focused on assessing attenuation systems for landing on soil, sand, and clay surfaces [1-3]. However, the land-landing capability was abandoned in favor of a water splashdown, similar to Mercury and Gemini [4-5]. Other studies in addition to the water landing studies were assessments of the capsule response in the event of a pad abort [6]. 
In 2009 the Orion Project lacked the test data to anchor LS-DYNATM analytical model predictions for the crew module (CM) water landing loads. These loads were the largest structural mass design driver and were predicted by modeling the physical interaction of the heat shield and side wall structures during water landing. Because these loads drove the design, early verification was extremely important. However, water drop tests were not planned until later in the program. To reduce the risk associated with using an unverified model for loads, a series of tests was undertaken for load verification. Specifically, the NASA Engineering and Safety Center (NESC) sponsored an effort to modify an existing full-scale boilerplate CM, referred to as the MAX Launch Abort System (MLAS), to use it as a pathfinder for the Orion Project water drop tests. Data from these tests were intended to provide an early assessment of the model adequacy.

Recall that Apollo water landing studies built on the extensive experience of the Mercury and Gemini programs. A wide range of experimental parameter studies, using subscale test articles, was conducted to evaluate the Apollo capsule performance. A primary interest of these studies was to understand the effect of landing attitude and longitudinal or horizontal velocity as well as heatshield flexibility on capsule response [7-11]. In addition, numerous full-scale tests were conducted to assess the capsule performance on more flight-like vehicles, e.g., References [1214]. Finally, based on knowledge of probable landing conditions, a design envelope was established [15]. Although the ORION project has benefitted tremendously from this work, the need for verification of computational models has not diminished, and must be undertaken to reduce program risk.

In this paper, data from two capsule water impact tests with the MLAS CM are used to compare measured pressures with predictions. However, comparisons are conducted using newly developed metrics that consider uncertainty and model adequacy from a multi-dimensional viewpoint. In fact the main focus of the paper is to show the applicability of these metrics when used with the pressure data. As a byproduct of the application of the metrics, the approach fosters the use of sensor placement algorithms to ensure that the data collected are adequate for model assessments. Because the placement approach was developed after the tests were 
completed, only analytical results are presented for illustration purposes. The paper organization is as follows; a test description is presented first, followed by calibration metrics, results from the application of the metrics to the test data, analytical sensor placement studies, and concluding remarks.

\section{Test Description}

MLAS test, analysis, and data evaluation were conducted in two phases over a two-year period. The first phase in February 2010 comprised 18 vertical drops at four water entry conditions. Tests used a crane at the littoral test facility at the U. S. Army’s Aberdeen Test Center (ATC) in Maryland, to position the capsule. A picture of the test article and a drop test are shown in figure (1). On-board instrumentation included accelerometers, inertial measurement units, and heat shield pressure sensors and strain gages. In addition, photogrammetric targets on the CM surface were tracked using high speed cameras to determine the CM position and attitude during the water entry.

For the second phase of testing the CM was modified with an expanded number of heat shield and side wall pressure sensors and internal strain gages on the primary structure load path. Thirty-eight drops at 21 conditions were completed at ATC in March 2011 with vertical velocities ranging from 135 to $440 \mathrm{in} / \mathrm{sec}$ and pitch angles from 0 to 40 degrees. Of all the data collected only tests with impact velocity of $300 \mathrm{in} / \mathrm{sec}$ and impact angles 30.6 deg and 21.5 deg were selected and used in this study, because repeated test data were available.

The LS-DYNATM [16] model then generated accelerometer, pressure, and strain predictions at key test conditions. The locations for the accelerometers and pressure transducers are shown in figure 2. Work here is focused on pressure data because that was the initial focus of the program.

\section{MLAS CM Boilerplate Model Description}

An LS-DYNA finite element structural model of the MLAS CM structure, figure (3a), was generated by personnel at NASA Ames Research Center starting from the CAD geometry. To stiffen the test article, engineers reinforced the core by adding an additional structure referred to as the "egg-crate”, see figure (3b). Most of the structure (ex. skin, ribs, heat-shield and 
stiffeners) was modeled using shell elements. However, concentrated masses were modeled using ballast connected by rigid links. Materials models in LS-DYNA used a Piecewise_Linear_Plasticity (material 24) formulation for all structural materials. The heatshield was constructed from ASTM A 516 grade 70 and the crew module was ASTM A572-50, except for the top cover assembly, removable hatch cover, CG sensor beam, and bracket which were aluminum. The characteristic length of the finite elements making up the model ranged from 3" to 0.5 ”. Water and vacuum volume domains were created and bias-mesh using 8-node solid elements with an ALE (Arbitrary-Lagrange-Eulerian) multi-material element formulation [16]. Dimensions for the water and vacuum meshed volumes are 240" x 240" x 48” and 240" x 240 ” x 12", respectively, with an element size of 1.0 " x 1.0” x 1.0" near the impact region. Finally, a penalty coupling method was used for modeling the fluid structure interaction (FSI) with a coupling pressure versus penetration curve shown in figure (4).

\section{Pressure Time History Analysis}

The analysis of time history pressure data from water impact tests must be guided by the way the data are used during the model calibration. For design, peak pressure values are often considered as adequate validation metrics used when judging simulation results. Although peak values often drives structural design, proper model calibration also needs to capture the spatially dependant load variations over the surface of the test article. Because oftentimes high frequency data are collected during tests, time independent calibration metrics can provide alternate evaluation tools to compare models. These metrics, initially developed for impact problems, are discussed next.

\subsection{Time Domain Calibration Metric-1}

Calibration metrics provide a mathematical construct to assess the fitness of a model in a quantitative manner. Work by Oberkampf [17-18] and Schwer [19] set forth scalar statistical metrics ideally suited for use with time history data. Metrics in terms of mean, variance, and confidence intervals facilitate assessment of experimental data, particularly when probability statements are sought. For our problem, instead of using response predictions at a particular point as a metric, a vector 2-norm (magnitude of vector) of the system response is used instead. An important benefit of using this metric is that it provides for a direct measure of multidimensional closeness of two models. In addition, when tracked as a function of time, closeness is quantified at each time step. 
Because parameter values are uncertain, statistical measures of the metric need to be used to conduct assessments. With limited information about parameter variations a uniform distribution function was selected and used to represent the parameter uncertainty. This parameter uncertainty model is used to create a family of $N$ equally probable parameter vectors with their corresponding responses, where $N$ is arbitrarily selected. From the perspective of a user, it is important to know the probability of being able to reconcile test with predictions, provided that both the model and parameter uncertainty model are appropriate. To this end, let $Q(t, p) \triangleq\|v(t, p)\|_{2}$ be a scalar time varying function, in which the vector $v$, constructed from multiple sensors, is used to compute the 2-norm of the response at time $t$. Furthermore, let $\underline{\sigma}(t)=\min _{\forall p} Q(t, p)$ be the minimum value over all parameter variations, and let $\bar{\sigma}(t)=\max _{\forall p} Q(t, p)$ be the maximum value. Using these definitions and $N$ LS-DYNA solutions corresponding to equally probable parameter vectors, a calibration metric can be established to bound the probability of test values falling outside the analysis bounds as;

$$
M_{1}=\operatorname{Pr} o b\left(\underline{\sigma}(t)<Q_{e}(t) \cap Q_{e}(t)>\bar{\sigma}\right)<<1 / N
$$

In here, $Q_{e}(t)$ is the 2-norm of measured experimental responses. Certainly, for cases with data

from multiple tests, $Q_{e}(t)$ must be replaced by its lower $Q_{e}(t)$ and upper bound $\bar{Q}_{e}(t)$. The arbitrary parameter $N$ controls tightness of the bounds and determines the number of LS-DYNA solutions required.

The use of norms, although convenient, tends to hide the spatial relationships that exist among responses at different locations in the model. In order to study the spatial multi-dimensional dependency explicitly, a different metric is needed. Before presenting the multi-dimensional metric, some needed preliminary time domain decomposition steps are discussed.

\subsection{Orthogonality Metric-2}

Data from either test or analysis is often collected at fixed time intervals for multiple sensors. For validation of models to predict loads from pressure pulses, it is preferable to collect data over the entire surface being affected. Unfortunately, practical constraints often limit the amount of data 
collected to smaller regions of the test article. Given $n$ samples of the response sampled at $\Delta T$, the time history can be decomposed as

$$
\left\{\begin{array}{c}
Y \\
Y_{e}
\end{array}\right\}=\left[\begin{array}{cccc}
y(0) & y(\Delta T) & \cdots & y(n \Delta T) \\
y_{e}(0) & y_{e}(\Delta T) & \cdots & y_{e}(n \Delta T)
\end{array}\right]=\left[\begin{array}{c}
\Phi \\
\Psi
\end{array}\right] \Sigma G
$$

The rightmost equality of Eq. (1.2) is obtained from singular value decomposition (SVD) of the time history data. Data is divided into $m$ measurements stored in $Y$ and $q$ unmeasured values stored in $Y_{e}$. In this decomposition, the basis matrices $\Phi \in \mathbb{R}^{m \times h}$ and $\Psi \in \mathbb{R}^{q \times h}$, referred to as basis or impact shapes, contains the time independent spatial distribution of sensors, whereas $G$ $\in \mathbb{R}^{h \times n}$ contains time variations, and $\Sigma$ contains the non-zeros singular values $\left\{\begin{array}{lllll}\sigma_{1} & \sigma_{2} & \cdots & \sigma_{n}\end{array}\right.$. From Eq. (1.2), one can solve for the unmeasured responses in terms of the measured values as

$$
Y_{e}=\Psi\left(\Phi^{T} \Phi\right)^{-1} \Phi^{T} Y
$$

After examining Eq. (1.3), it should be apparent that sensor placement plays an important role in our ability to predict unmeasured responses $Y_{e}$. For example, if the matrix $\Phi$ contains rows/columns which are linearly dependent (i.e. sensors are placed poorly), Eq. (1.3) becomes numerically ill-conditioned and cannot be used to estimate the response $Y_{e}$. For this reason, sensor placement for this class of problem must be guided by the numerical conditioning of Eq. (1.3). Furthermore, because some impact shapes contribute more to the overall response than others, sensors must be placed to properly recover the most important impact shapes. Importance of the $i^{\text {th }}$ impact shape is quantified in terms of its contribution to the total response as

$$
\delta_{i}=\sigma_{i} / \sum_{l=1}^{n} \sigma_{l}
$$

In this decomposition, the sum total of the impact shape contribution adds up to one.

With the decomposition approach just discussed, one can now develop a multi-dimensional metric to compare models. Specifically, the impact shape vectors computed with Eq. (1.2), provide a means to compare models using time invariant metrics like those normally used in classical modal test. Impact shapes are ideally suited to compare analytical models with test using orthogonality. Numerically, the orthogonality metric is computed as;

$$
M_{2}=\widehat{\Phi}^{T} \widehat{\Gamma}
$$


where $\widehat{\Phi}$ is sized $m \times h$ with $h$ measured impact shapes at $m$ locations and $\widehat{\Gamma}$ sized $m \times h$, are shapes computed using simulation data. Both $\widehat{\Phi}$ and $\widehat{\Gamma}$ have been normalized such that $\widehat{\Phi}^{\mathrm{T}} \widehat{\Phi}=I$ and $\hat{\Gamma}^{T} \hat{\Gamma}=I$. Because individual impact shape vectors are stacked column-wise, the orthogonality metric $M_{2}$ is sized $h \times h$ with diagonal values corresponding to the vector projection numerical value. If vectors are identical then their projection equals 1 . Consequently, when evaluating models, multi-dimensional closeness is judged based on similarity of impact shapes and shape contributions.

\subsection{Prediction Error Metric-3}

Two metrics have been presented to assist the model calibration process; $M_{1}$ quantifies the probability of being able to reconcile model with test data, whereas, $M_{2}$ addresses model closeness from a multi-dimensional viewpoint. Although both $M_{1}$ and $M_{2}$ measure our predictive capability between test and analysis, a third metric is needed for this class of problems for the sole purpose of conducting sensor placement. This metric considers the error resulting from predictions made using data from a reduced set of sensor locations. Intuitively, one would expect certain sensor locations to provide a better set of system responses when compared to other locations. To quantify this, the prediction error metric is defined as

$$
M_{3}=\left(\widehat{Y}_{e}-Y_{e}\right)^{T} W\left(\hat{Y}_{e}-Y_{e}\right)
$$

In here, the quadratic prediction error metric $M_{3}$ is weighted by an arbitrary diagonal weighting matrix $W$. This weighting matrix is convenient for cases where the user needs to reject sensor locations for other reasons. Since this metric uses only simulation data, it is simple to evaluate various candidate sensor sets. To evaluate Eq. (1.6) one uses the exact values for $Y_{e}$ from simulation results and estimated values (using Eq. (1.3)) for $\widehat{Y}_{e}$. The best sensor set will produce the smallest $M_{3}$. In order to obtain a candidate sensor set, combinatorial optimization algorithms [20] are ideally suited for the problem. Results later in the paper demonstrate this approach and how it compared to the baseline sensor set.

\section{Results using Metrics with Measured Pressure using Metrics}

At this point, our attention is turned to study the pressure data from tests with impact velocity of $300 \mathrm{in} / \mathrm{sec}$ and impact angles $30.6 \mathrm{deg}$ and $21.5 \mathrm{deg}$. These two cases will be referred to as T300-30 and T300-21, respectively. To demonstrate the application of the metrics discussed in 
Section 5.1, the sensor set was restricted to 33 pressure sensors. These 33 sensors are all within a 66 inch radius centered at the heat shield impact point, located at $x=779.82, y=-1.25$, and $z=$ 96.91. Although this sensor set is not the full set, many other sensors were left out because they did not see pressure values until after $50 \mathrm{msec}$, which is the time window considered here. For the T300-30 condition, data from 7 tests were used, whereas for the T300-21 data from 8 tests were used. Recall that metric $M_{1}$ is used to assess test variation where the maximum and minimum singular value bounds are computed using 33 pressure sensors. Figure 5 shows the test uncertainty bounds (i.e., $\bar{Q}_{e}(t)$ and $\underline{Q}_{e}(t)$, solid-blue) for T300-30. To construct the tests bounds, the magnitude norm using 33 pressure sensors is computed for each of the 7 tests first. Then the maximum of all pressure maxima $\bar{Q}_{e}(t)$ and minimum of all pressure minima $\underline{Q}_{e}(t)$ are collected and plotted as a function of time. Note that the observed variation (represented by the difference between the upper and lower bounds) from 7 very similar tests is large. Because data from 7 tests fall within these bounds, it is reasonable to assume that the probability of observing new test data outside these bounds is less that $1 / 8$.

To contrast the uncertainty in the experimental data to predictions from the analytical model, the LS-DYNA model was executed multiple times assuming that the impact velocity, pitch, and yaw angles were uncertain. Table 1 shows the nominal conditions for T300-30 along with selected parameter upper and lower bounds. Uncertainty data reported by the test group was used to determine initial parameter bounds but in the end, the bounds used were significantly larger than those provided by the test group. With this uncertainty model, parameters are assumed to be equally likely between bounds, and as such parameters were varied to generate 28 impact conditions near the T300-30 nominal case. As with test data, bounds for predicted pressure data are collected from $N=28$ simulations. Results for the analytical bounds ( $\underline{\sigma}$ lower bound and $\bar{\sigma}$ upper bound) are overlaid to the test data in figure 5; dashed red lines correspond to analysis. From metric $M_{1}$ (shown in figure 5) and the selected uncertainty model, the probability of predicting analytical results outside the analytical bounds is less that $1 / 28$. Hence, with the current LS-DYNA model and uncertainty models, it is difficult to explain test observations outside the analysis bounds. This is in spite of the fact that the parameter bounds used in this study were significantly larger than those reported by the test group. Results for metric $M_{1}$ and 
T300-21 are shown in figure 6 using 8 tests and 35 LS-DYNA runs. Because with this model each run takes over 28 hours (using a 4 processor $3.3 \mathrm{GHz}$ desktop computer), the number of LSDYNA runs executed is constrained by available time. Parameters for T300-21 are shown in Table 2. As with T300-30 cases, analytical uncertainty bounds do not enveloped the test results and therefore, with this model it is difficult to reconcile test with analysis.

Another way to compare test results to analysis is by using the orthogonality metric $M_{2}$. In here, time invariant impact shapes or basis vectors computed using pressure test data are compared to analysis, Eq. (1.5). Results for test 1 of the T300-30 series are shown in figure 7a, and results for test 7 are shown in 7b. Black squares correspond to impact shapes with high orthogonality values between test and analysis. About 9 impact shapes exhibited orthogonality values greater than 0.6 for test 7 versus only 5 for test 1 . Consequently, results from test 7 (T300-30) are closer to the analysis model. Across the top of the orthogonality plot are printed the test impact shape contribution to the overall response, whereas the ordinates show values from LS-DYNA. For example, from figure $7 \mathrm{a}$, the first test impact shape contributed $14 \%$ as compared to $19 \%$ from LS-DYNA. Results for the T300-21 are shown in figure 8. Figure 8a compares the orthogonality values for test 8 whereas figure $8 \mathrm{~b}$ compares results for test 4 . Clearly, test 4 for the T300-21 shows better agreement between test pressures and LS-DYNA.

\section{$7 \quad$ An Approach for Pressure Sensor Placement}

Because the sensor placement approach in the following was not used during test, it is presented last for readers interested in more rigorous methods for sensor placement. When optimal sensor placement is performed as part of the pre-test analysis, placement is driven by the test goals. If model calibration is the goal, sensor placement must focus on providing information to properly evaluate the established calibration metrics. For example, when the orthogonality metric $M_{2}$ is used, if sensors are not strategically placed, it is difficult to distinguish between basis vectors. Moreover, if our goal is to conduct pressure predictions, prediction error must also be considered during placement. Our approach uses combinatorial optimization to select a fixed number of sensors from a large number of probable locations. To make the problem more tractable, the area for sensor placement was confined to a circular sector near the impact point. 


\subsection{Post-Test Pressure Sensor Placement Study}

Placement of pressure sensors for the MLAS CM boiler plate test was conducted based on best engineering practices. Specifically, sensors were spaced uniformly near the expected impact point, with increased spacing as one moved away from impact. Pressure sensors at 60 locations, as shown in figure $2 b$, were selected and used during test. Because of symmetry considerations, the bulk of the sensor set located during test were concentrated on the lower right quadrant of figure $2 \mathrm{~b}$. From test conditions, the impact point was expected to be near the $\mathrm{z}$-axis center line, and it moved along that line depending on the impact angle. For our placement study, node 5017879 with coordinates $(779.82,-1.25,96.91)$ was selected as the impact center and all elements within a radius of 66 inches were considered for sensor placement. This area provided for a total of 2148 possible locations where pressure readings could be reported from quadrilateral elements in the region. Figure 9 shows a projection onto the $y$-z plane of the heat shield augmented sensor set (2148 location) using blue (+), with red circles (o) showing the pressure sensors locations used during test. Only 33 of the 60 sensors used during test are within the 66 inch radius used in this study. For comparison purposes, it is assumed that only 33 sensors can be relocated to see if the metrics can be improved using an alternate sensor placement technique.

\subsection{Analytical Basis Vectors for MLAS CM Water Impact Simulation}

Because orthogonal decomposition for this class of problems is not performed routinely, it is important to understand the spatial time independent information captured in the basis vectors computed using Eq. (1.2). In particular, if characteristic patterns appear from pressure data, these patterns can help tremendously in placing sensors and more importantly in predicting the response at other locations. For this reason, a set of basis vectors was computed using simulated data and all 2148 possible sensor locations. Figure 10 shows contour plots of the heat shield region in the $y$-z plane for the first 4 basis vectors using simulated time histories. The contours are constructed using the numerical values of the basis vectors plotted along the $y$-z plane for the heat shield section. Note that these characteristic patterns are similar to wave front propagation pulses. In addition to the patterns, numerical values for the contribution of the first 4 basis vectors to the overall response are shown. These values indicate that the $1^{\text {st }}$ basis contributes $13.5 \%$ to the overall response whereas basis number 2 contributes $8.35 \%$. Because the sum total contribution from 4 bases is less that 33\%, it should be apparent that many more basis vectors 
participated in the overall response. As already mentioned, studying these patterns is very helpful to understand qualitatively the complexity of a pressure pulse, unfortunately, what is seen with a reduced set of sensors is far less. For example, figure 11 shows results for the first two basis vectors as seen through the 33 pressure sensors used in the simulated test. Certainly, it is extremely difficult to discern patterns but more importantly, the computed contribution is significantly different when compared to the full set of possible sensor locations.

\subsection{Optimum Pressure Sensor Placement}

This section discusses an optimization based approach to place pressure sensors for this class of problems. The reader is reminded that this is strictly for illustration purposes because the approach was not implemented for testing. Nonetheless, the approach that follows recognizes that sensor placement must serve a dual role: 1) to calibrate the analytical model at locations where measurements exist, and 2) to allow one to make predictions at other locations. Often times these two roles are thought to be the same, but in fact that is not necessarily the case. For example, if there is local behavior captured in the model but not measured, then it is difficult to recover such behavior from measured data. In Section 5.2 an approach was discussed to perform response predictions using a reduced set of observation points and the analytical model. Effectively, the analytical model is used to provide interpolation functions to make predictions at other locations. Also discussed was the prediction error metric $M_{3}$ to quantify the error incurred when conducting pressure predictions at other locations using only data from a reduced set of observation points. If sensors are placed properly, then predictions made throughout the surface will have minimum error.

For the MLAS CM boiler plate test pressure sensors were placed (as shown in figure 2b) without regard to the ability to make predictions at other locations. Although placement using a heuristic approaches like the one used with MLAS can provide quality pressure data (as will be shown later), it hinders the ability to make predictions at other locations. To demonstrate this problem, a combinatorial optimization algorithm was used with simulated data to place 33 sensors. In here the sensor count was kept as in the test to maintain similar coverage. The optimization problem was tasked to choose 33 pressure sensor locations out of the 2148 possible locations while minimizing the prediction error metric $M_{3}$. Figure 12a shows again the heat shield projected 
onto $y$-z plane with the baseline sensors locations (o) and figure 12b shows the sensors placed using optimization (o). These results by themselves are not very revealing until one examines the prediction error. Figure 13a shows the normalized prediction error as a function of location when using responses from simulation at the baseline locations (figure 12a) to predict responses at all 2148 points. Certainly, at the 33 baseline locations the normalized prediction error is zero. Two aspects of the results should be apparent, 1) the normalized prediction error is very large $(>>1)$ in some regions, and 2) there are clusters of regions where the error is small. Areas where the error is small are near measured points. However, in general the normalized error, defined as $e=\max \left(y_{e}-y\right) / \max (y)$ where $y_{\mathrm{e}}$ is the prediction and $y$ is the exact value, is very large for many regions over the heat shield. In contrast, Figure 13b shows the normalized prediction error with sensors placed using optimization. For this case the normalized prediction error is three orders of magnitude smaller than results obtained using the baseline sensor set. Finally, to demonstrate the applicability of Eq. (1.3), figure 14 shows estimated pressures at two arbitrarily selected locations on the heat shield. Figure 14a shows time traces comparing pressure predictions (dashed) and the exact values (solid) at two different locations. Figure 14b show stars to indicate the location of these arbitrary points with respect to locations selected by the optimizer. Clearly, results shows that one is able to predict pressure responses at locations away from measured points. Nonetheless, the process can introduce significant numerical errors as evident in the prediction for pressure ID 5012903 in Fig. 14a.

\section{Concluding Remarks}

The paper discussed the application of newly developed calibration metrics to assess model adequacy in terms of probability of predicting the measured behavior and a multi-dimensional metric to measure model closeness. As a byproduct of implementing the metrics for model evaluation, a sensor placement approach was discussed and demonstrated to improve pressure predictions at points where measured data were not available. Unfortunately, the placement approach was developed after tests were completed and therefore could not be verified through test. Future testing should incorporate pre-test sensor placement as described in this paper to improve our ability to make pressure predictions at other locations. 
To study the effects of variations in impact velocities and impact angles analytically, variations in these conditions were used to create a family of pressure pulse predictions. These results provided envelopes for the expected pressure predictions from the analysis as well as probability values. Data from two MLAS CM boilerplate tests were superimposed onto the analytical envelopes and it was determined that test results could not be enveloped by the analysis. Hence, test results cannot be reconciled with the analysis using the current model. Without additional data no improvements to the model are possible. 
Table 1 Uncertainty parameters for T300-30 study

\begin{tabular}{|c|l|c|c|c|}
\hline No. & \multicolumn{1}{|c|}{ Parameters } & Nominal & Upper & Lower \\
Values & Bound & Bound \\
\hline 1 & Pitch Angle (deg) & 30 & 31 & 29 \\
\hline 2 & Yaw Angle (deg) & 0 & 0.4 & -0.4 \\
\hline 3 & Vertical Velocity (in/sec) & 300 & 302 & 293 \\
\hline
\end{tabular}

Table 2 Uncertainty parameters for T300-21 study

\begin{tabular}{|c|l|c|c|c|}
\hline No. & \multicolumn{1}{|c|}{ Parameters } & $\begin{array}{c}\text { Nominal } \\
\text { Values }\end{array}$ & $\begin{array}{c}\text { Upper } \\
\text { Bound }\end{array}$ & $\begin{array}{c}\text { Lower } \\
\text { Bound }\end{array}$ \\
\hline 1 & Pitch Angle (deg) & 21.5 & 22.5 & 20.5 \\
\hline 2 & Yaw Angle (deg) & 0 & 0.4 & -0.4 \\
\hline 3 & Vertical Velocity (in/sec) & 300 & 302 & 293 \\
\hline
\end{tabular}




\section{References}

1. Hartwig, H.H and Schmidt, J. L.: "Summary- Apollo Earth Landing System Impact Attenuation Trade-Off Study,” North American Aviation SID 62-897, July 1962.

2. Stubbs, S.M.: "Investigation of the Skid-Rocker Landing Characteristics of Spacecraft Models," NASA TN D-1624, April 1963.

3. Benson, H.E. and West, R.B.: "Results of Two One-Quarter Scale Apollo Model Impact Tests Utilizing Different Impact Attenuation Systems,” NASA TMX-65129, January 1965.

4. "Water Impact of the Mercury Capsule Correlation of Analysis with NASA Tests," Grumman Aircraft Engineering Corporation, NASA CR-55251, December 1962.

5. Thompson, W.C.: "Dynamic Model Investigation of the Rough-Water Landing Characteristics of a Spacecraft,” NASA TN D-3774, April 1967 (Technical Film Supplement L-940).

6. Reese, T. and Rose, J.D.: “Apollo Pad Abort Land Impact Tests at Kennedy Space Center,” NASA TM X-64365, April 1969.

7. McCullough, J. E. and Lands, J. F. Jr.: “Apollo Command Module Land-Impact Tests,” NASA TN D-6979, October 1972.

8. Baker, W. E., Westine, P. S., Garze, L. R., Hunter, P. A.: "Water Impact Studies of Model Apollo Command Module,” NASA CR-114949, August 1965.

9. Baker, W.E and Westine, P.S.; "Model Tests for Structural Response of Apollo Command Module to Water Impact,” AIAA Journal of Spacecraft and Rockets, 0022-4650, Vol. 4 No. 2, 1967.

10. Stubbs, S.M.: "Dynamic Model Investigation of Water Pressures and Accelerations Encountered During Landings of the Apollo Spacecraft,” NASA TN D-3980, September 1967. (Technical Film Supplement L-960).

11. Stubbs, S. M Hathaway, M. E.: "Effects of Bottom-Structure Flexibility on Water Landing Loads of Apollo Spacecraft Models,” NASA TN D-5108, March 1969.

12. “Final Test Report Water Impact Test 92 Boilerplate 28 (Drop 3)”, North American Aviation SID 66-35, April 1967.

13. "Final Test Report Water Impact Test 104 Spacecraft 2S-1 (Drop 2)”, North American Aviation SID 66-330A, April 1967.

14. "Study of Apollo Water Impact Final Report: Volume 4 - Comparison with Experiments," North American Aviation, NASA CR-92022, May 1967.

15. "Apollo Applications Program Preliminary Evaluation Data - Apollo Landing and Recovery Subsystem Analyses,” North American Rockwell, SID-69-244, April 1969.

16. Hallquist, John Q., “LS-DYNA Keyword User’s Manual,” Version 971, Revision 5.0, Livermore Software Technology Company, Livermore, CA, May 2010.

17. Oberkampf, W.L. \& Barone, M.F. (2006). Measures of Agreement Between Computation and Experiment: Validation Metrics. Journal of Computational Physics, 217, pp. 5-56, June 2006.

18. Oberkampf, W.L., Trucano, T.G., \& Hirsch, C. (2003). Verification, Validation, and Predictive Capability in Computational Engineering and Physics. SAND 2003-3769, February 2003.

19. Schwer, L.E. (2007). Validation Metrics for Response Histories: Perspectives and Case Studies. Engineering with Computers, Vol. 23, No. 4, pp. 295-309, June 2007. 
20. Chipperfield, A., Fleming, P. J., Pohlheim, H. and Fonseca, C. M.: "Genetic Algorithm Toolbox for use with Matlab.” Technical Report No. 512, Department of Automatic Control and Systems Engineering, University of Sheffield, 1994. 


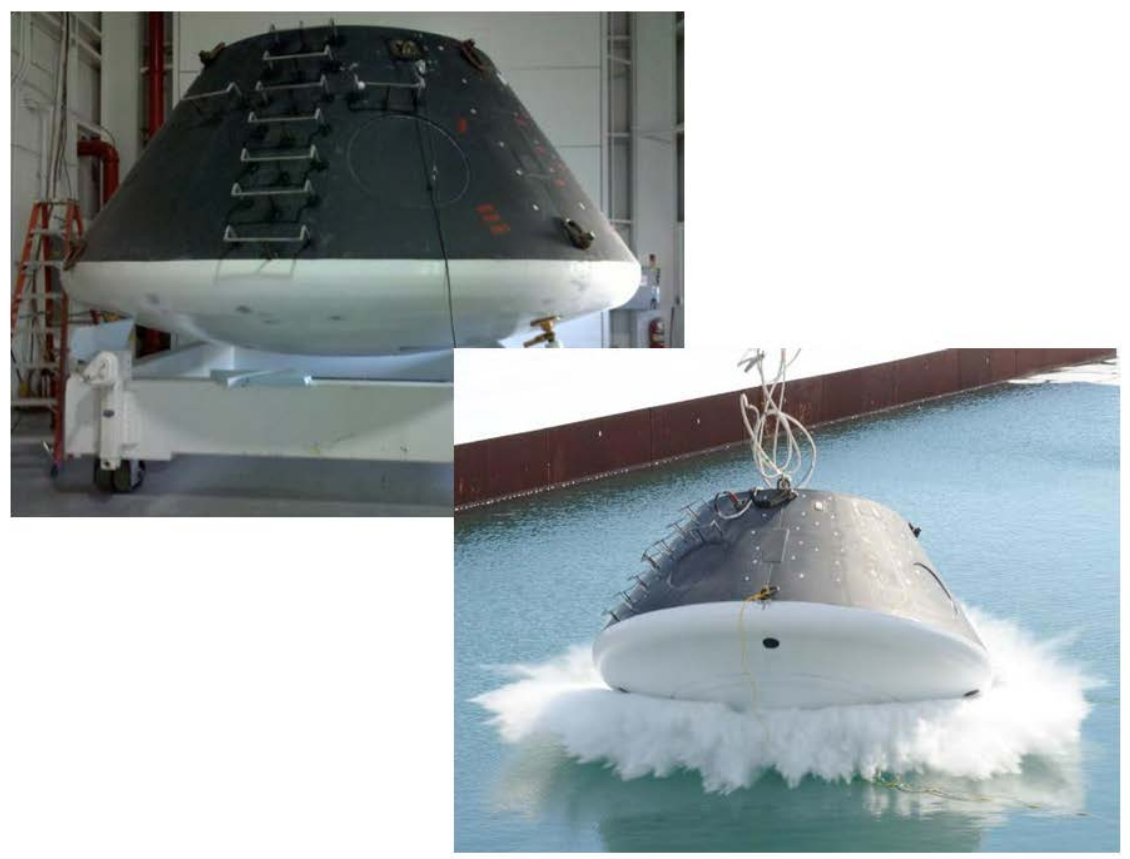

Figure 1. MLAS test article and water impact test at the U.S. Army Aberdeen test

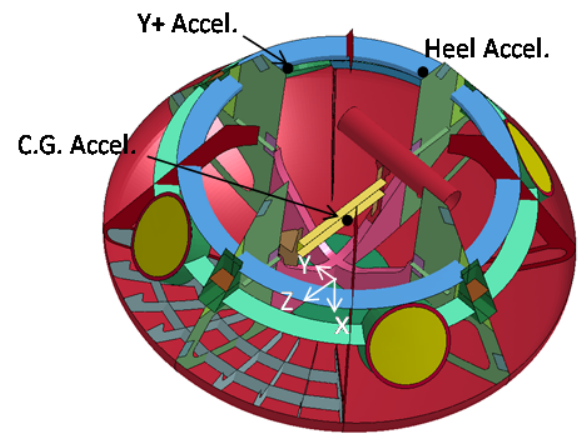

Figure 2a. Acceleration Sensor locations

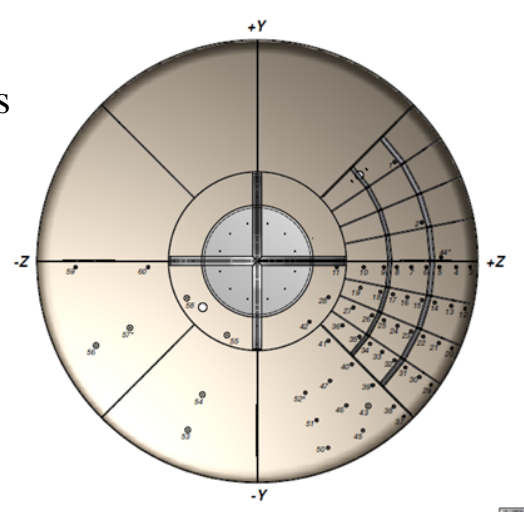

Figure 2b. Pressure sensor locations 


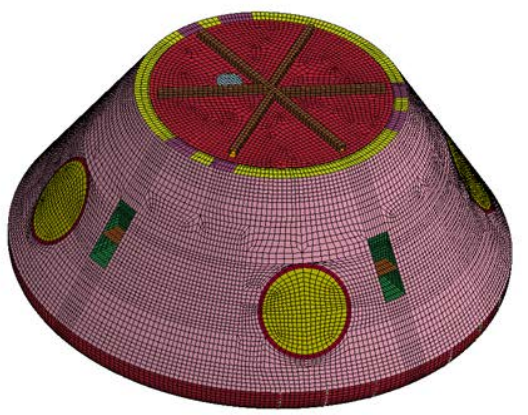

Figure 3a. MLAS FEM

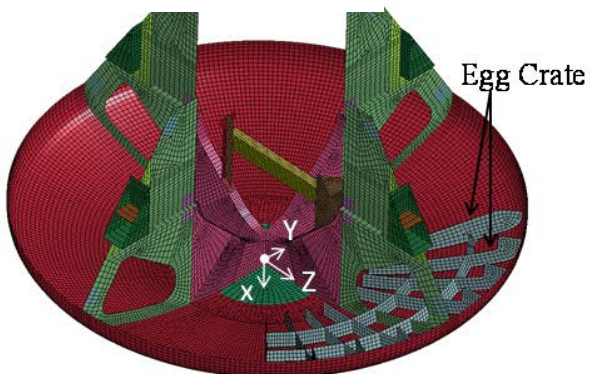

Figure 3b. Egg crate and local coordinate system

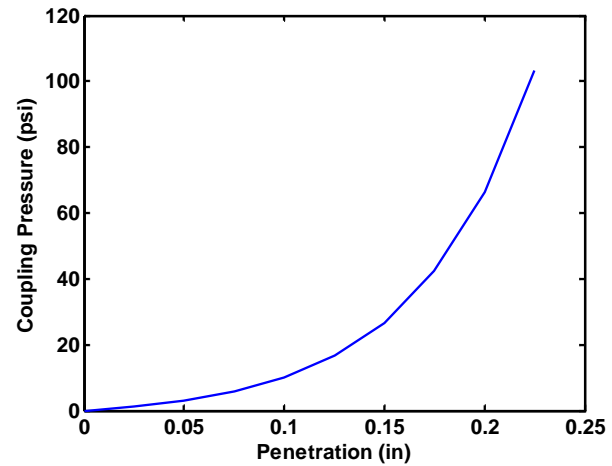

Figure - Fluid Structure Interaction (FSI) Coupling Curve

Figure 4. Fluid Structure Interaction (FSI) coupling curve 


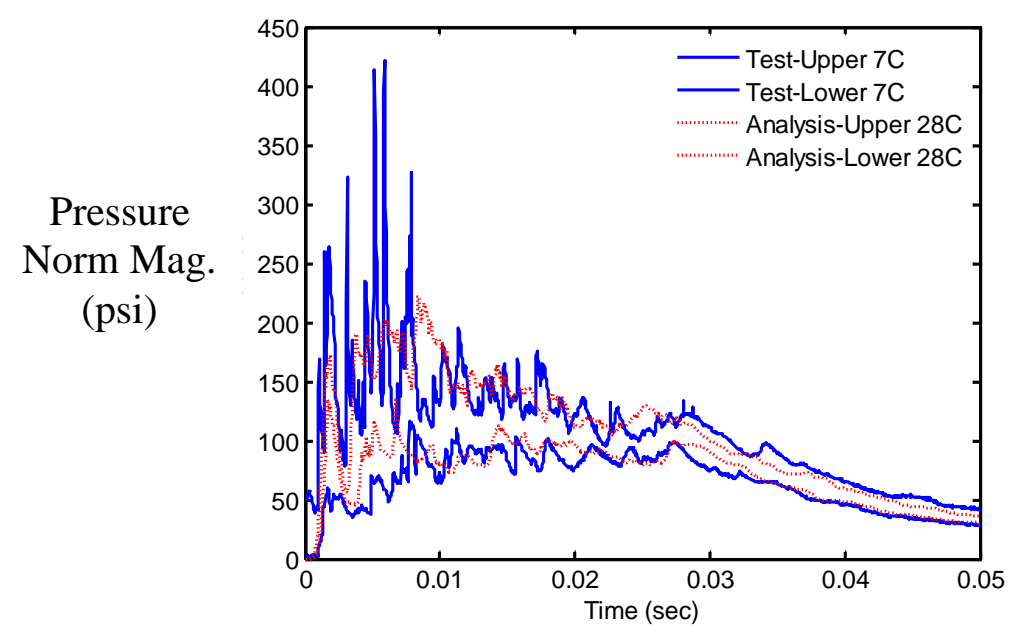

Figure 5. Pressure data uncertainty bounds for T300-30 (solid-blue) using 7 tests and uncertainty bounds for analysis (dashed-red) using 28 cases

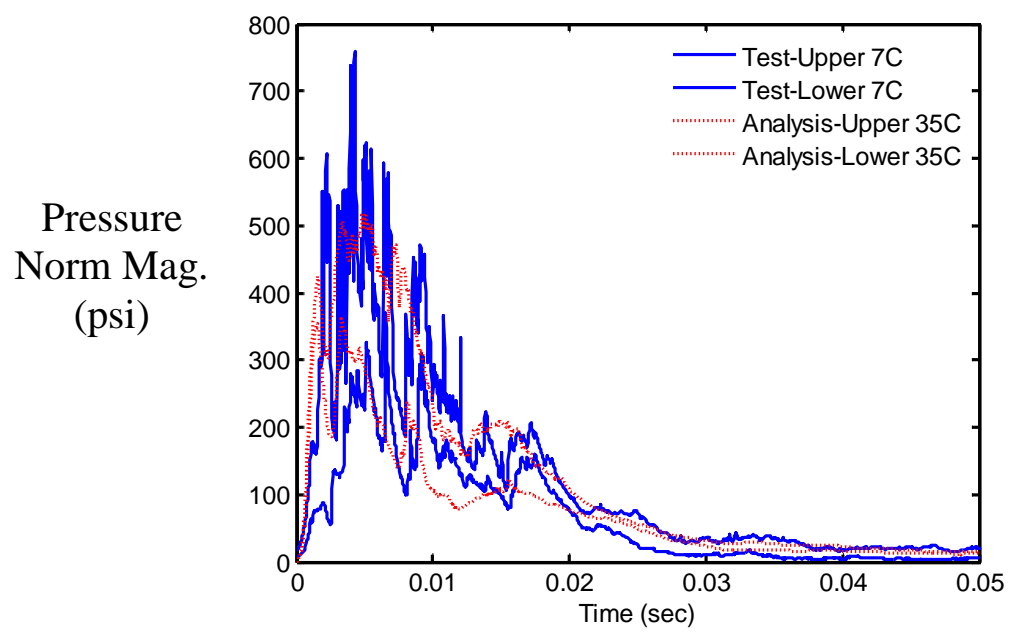

Figure 6. Pressure data uncertainty bounds for T300-21 (solid-blue) using 7 tests and uncertainty bounds for analysis (dashed-red) using 35 cases 
a) T300-30 Test 1

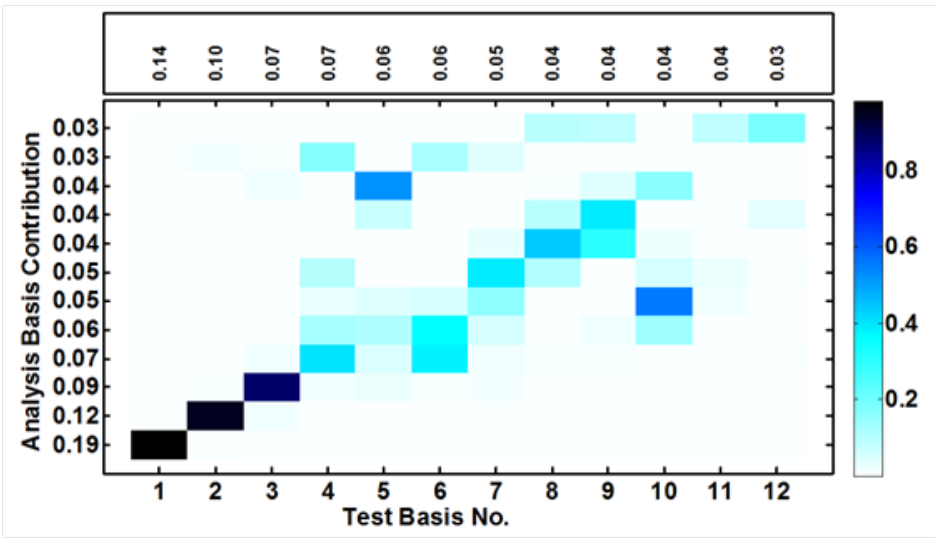

b) T300-30 Test 7

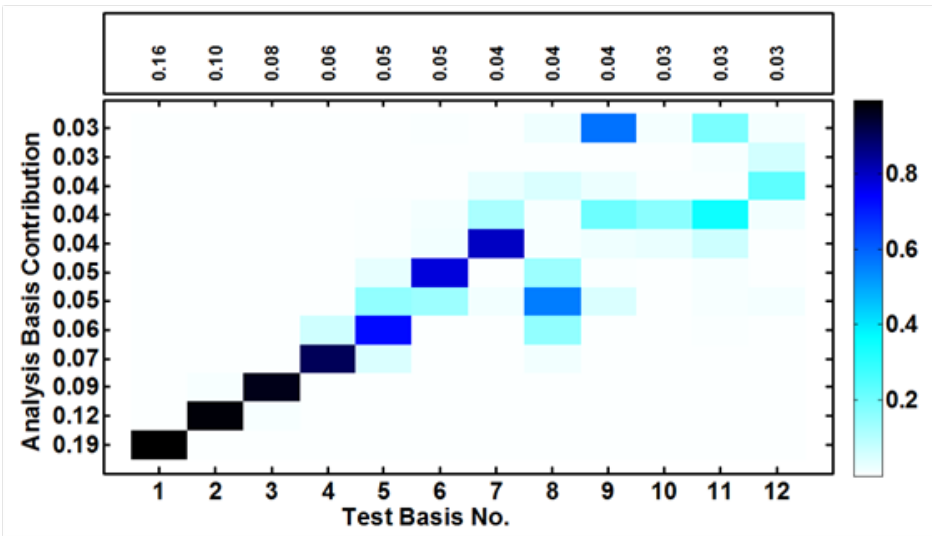

Figure 7. Orthogonality of measured and predicted impact shapes using pressure data for T300-30 
$\begin{array}{ll}\text { a) } & \text { T300-21 Test } 8 \quad \text { Test No. } 8 \text { vs DYNA Set } \\ \text { Test Basis Contribution }\end{array}$
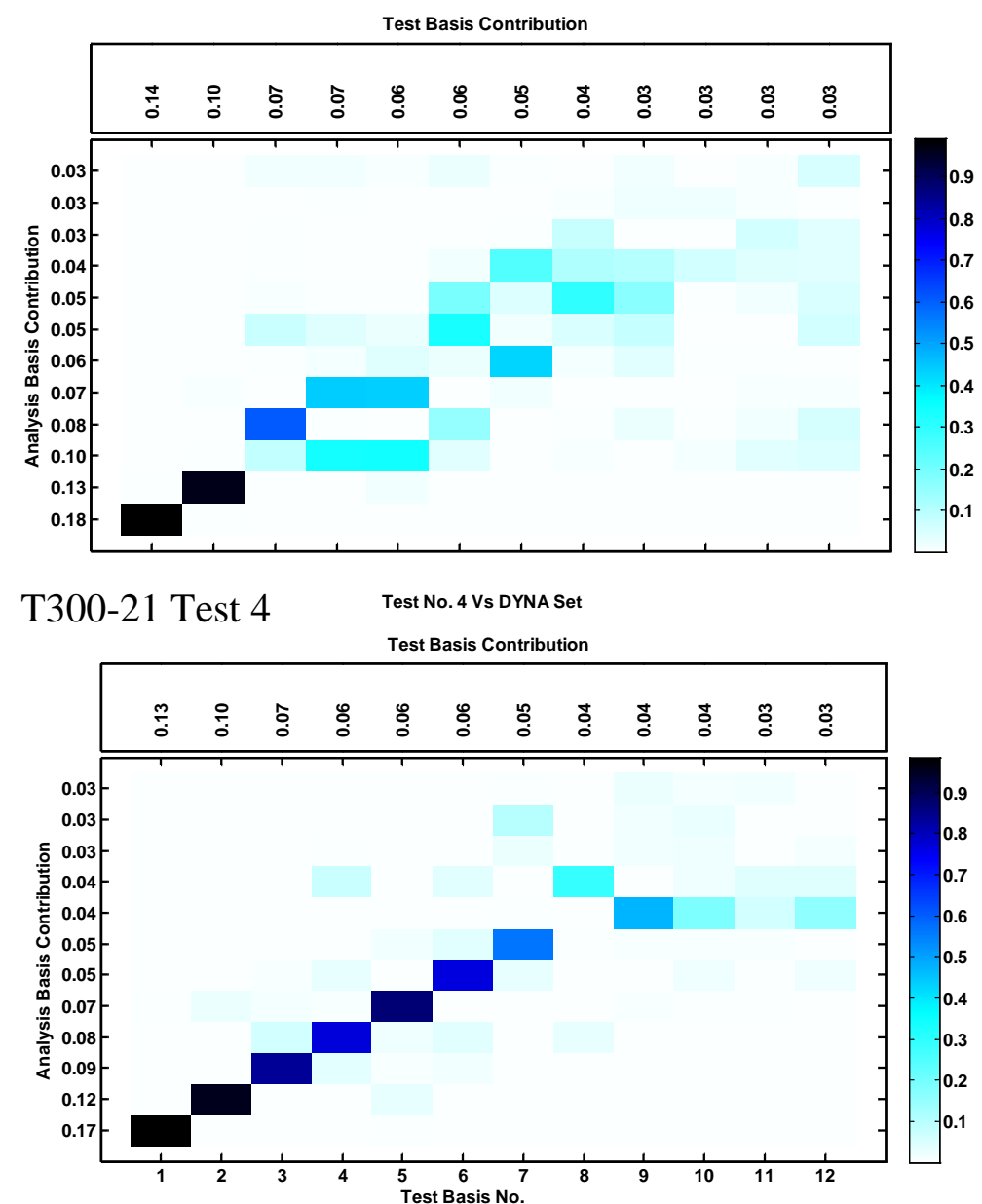

Figure 8. Orthogonality of measured and predicted impact shapes using pressure data for T300-21 test case 8 at the top and test case 4 at the bottom. 


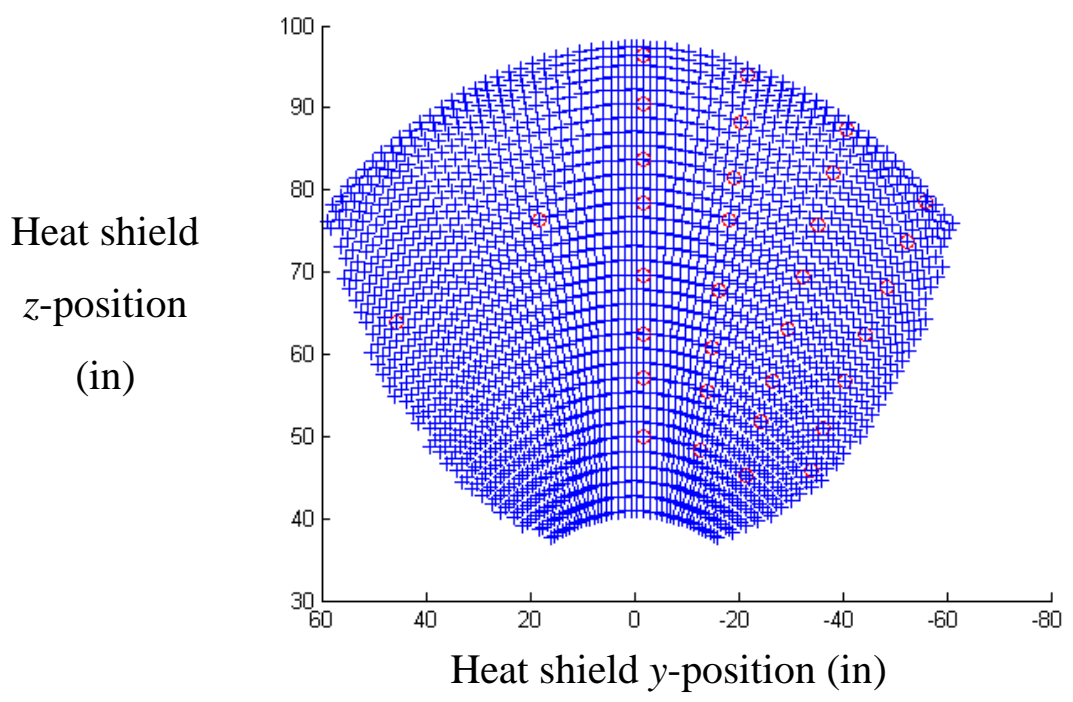

Figure 9. Augmented sensor set $(+)$ and test sensor (o) locations
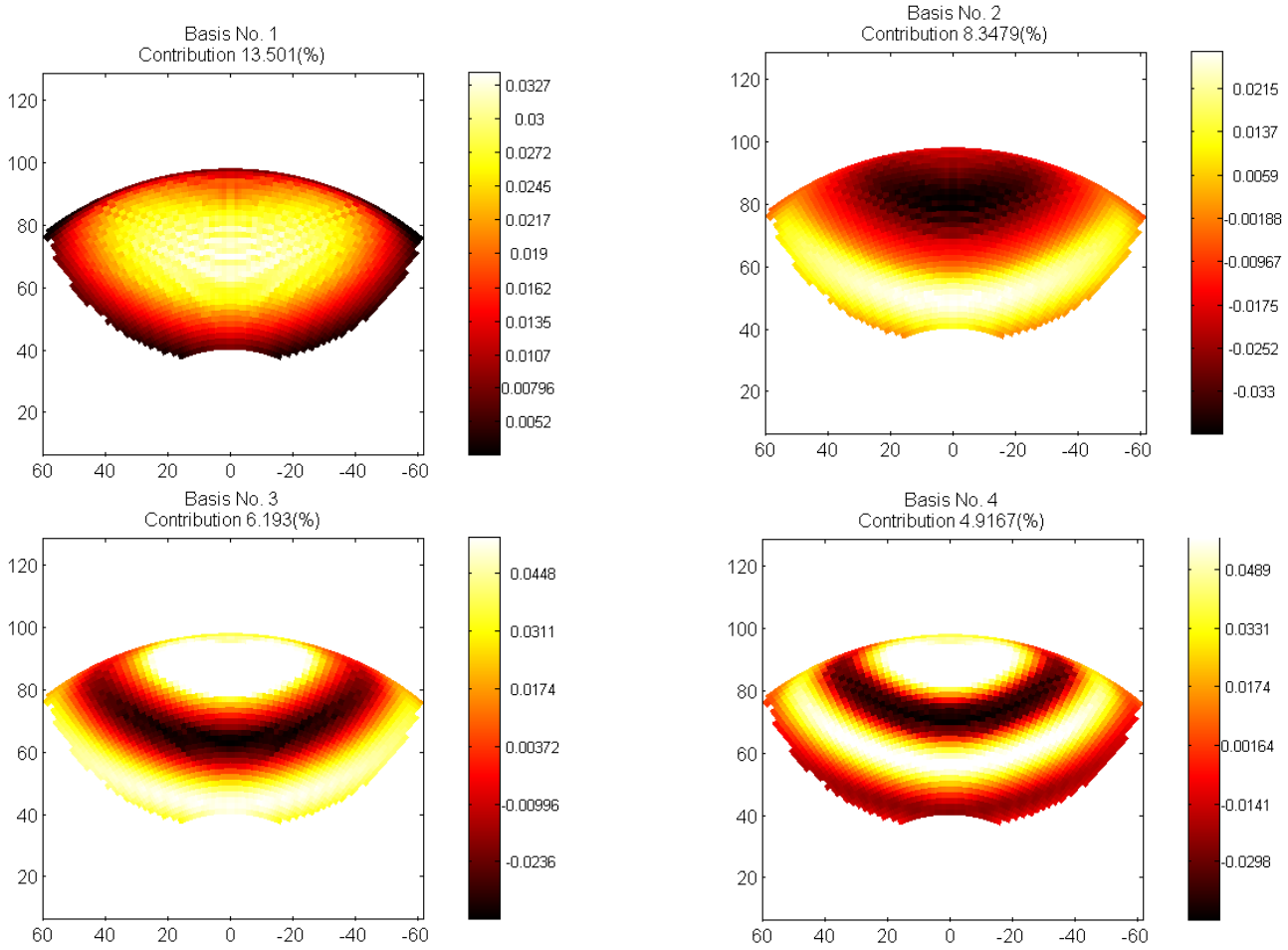

Figure 10. Contour plots of the first 4 basis vectors using pressure data and 2148 

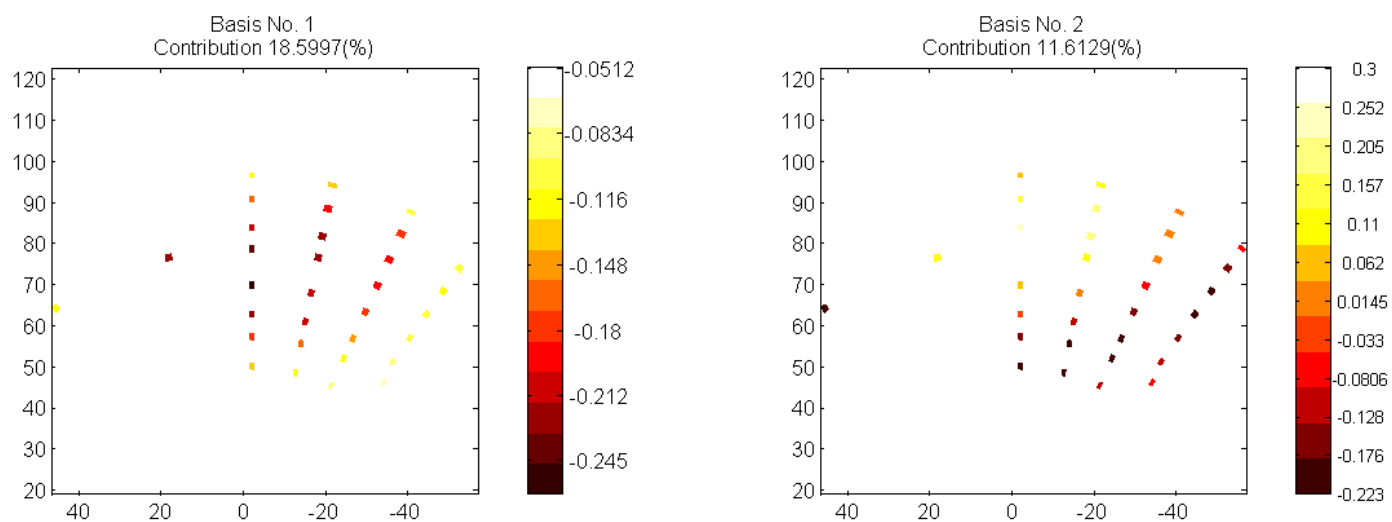

Figure 11. Contour for first two pressure basis vector using 33 measured locations
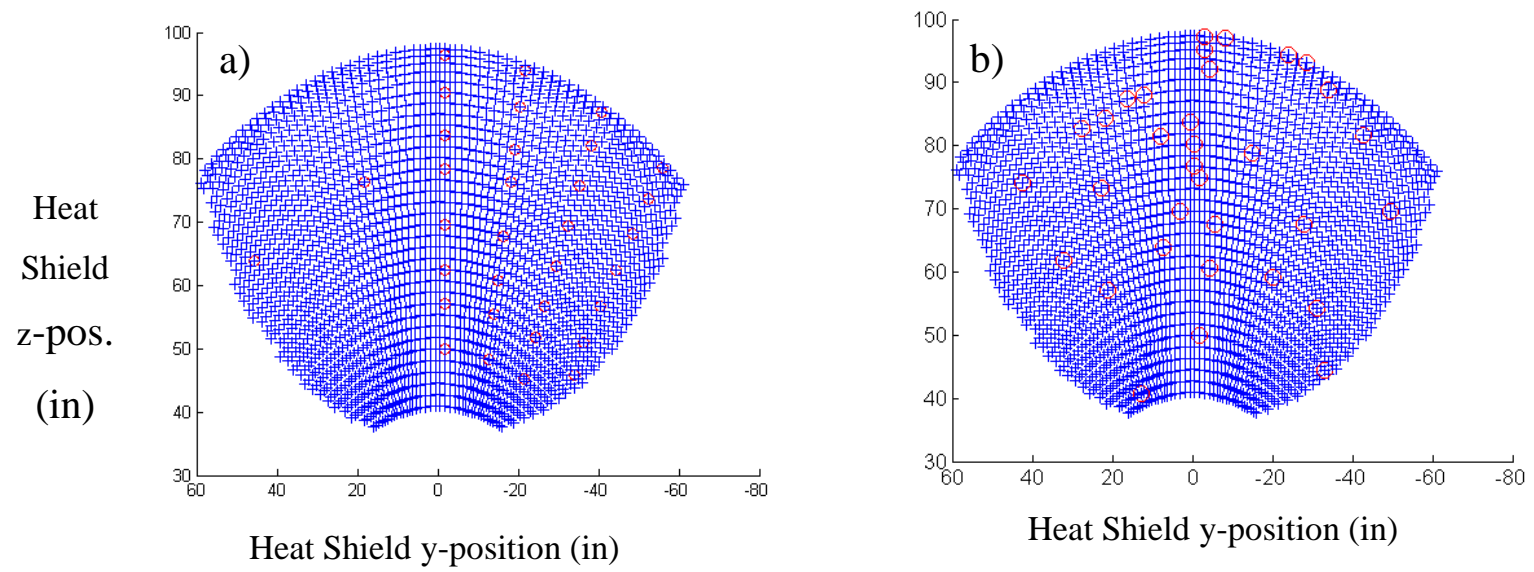

Figure 12. Comparison of baseline pressure sensor placement and optimized placement using prediction error
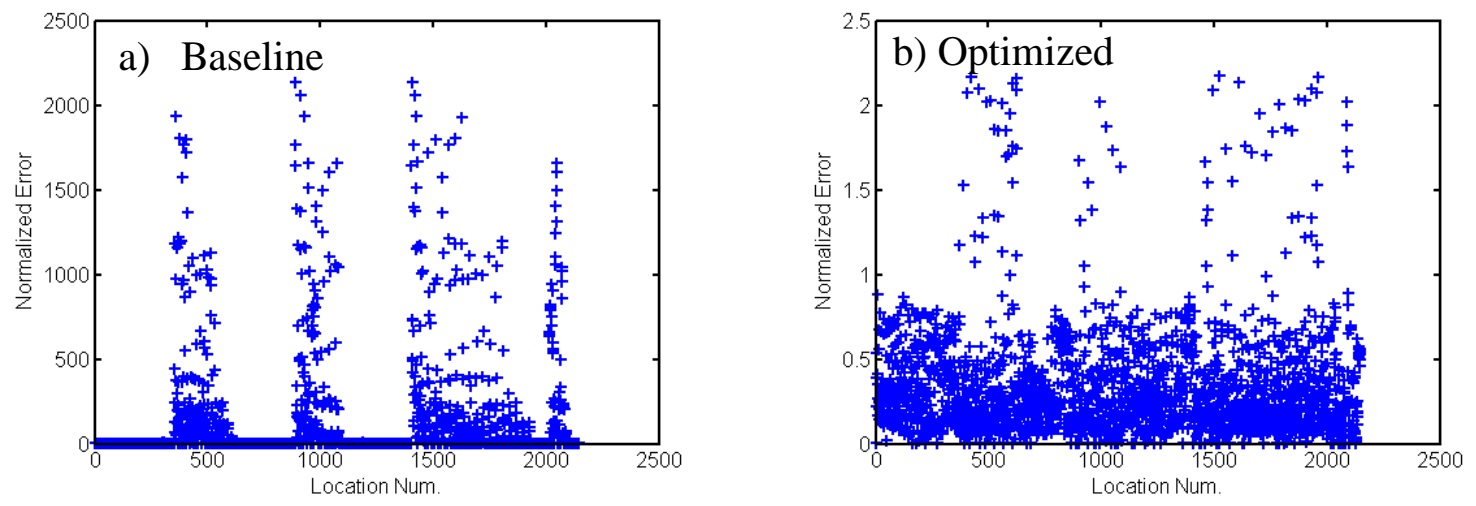

Figure 13. Comparison of normalized prediction error for pressure on the heat shield using baseline sensor placement and optimized sensor placement 

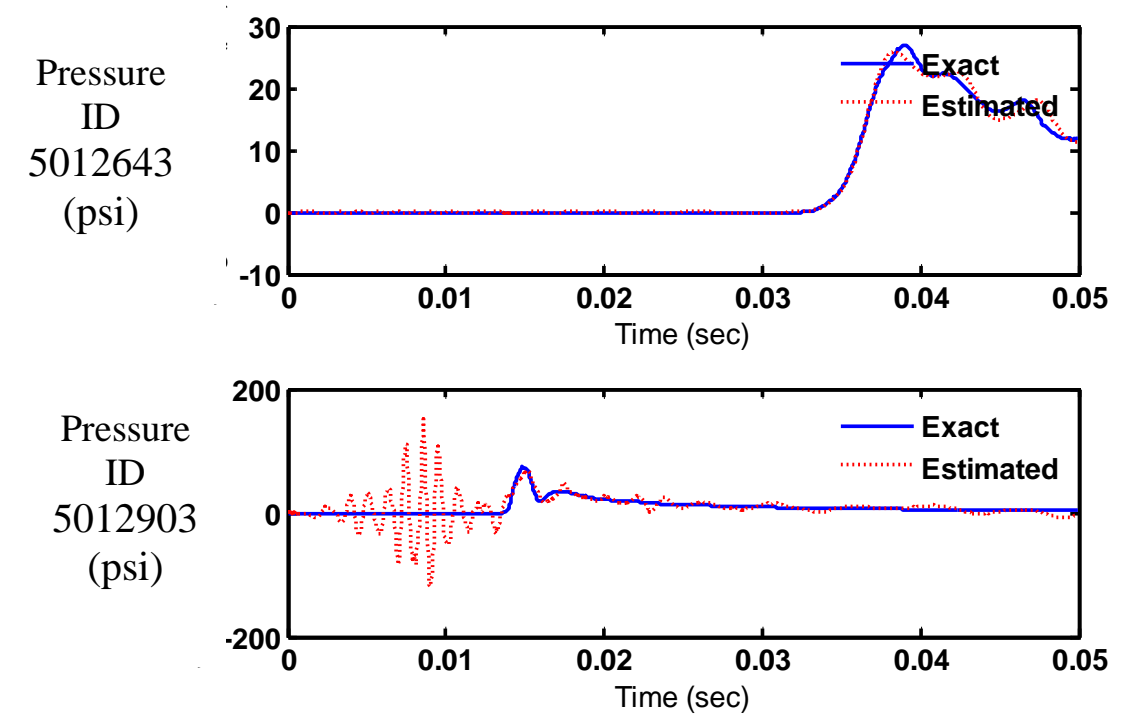

a) Pressure predictions



b) Pressure locations

Heat Shield $y$ (in)

Figure 14. Pressure predictions at unmeasured locations 